Z Herz- Thorax- Gefäßchir 2015 · 29:82

DOI 10.1007/s00398-015-1150-y

Online publiziert: 3. April 2015

(c) Springer-Verlag Berlin Heidelberg 2015

\title{
D.-S. Dohle ${ }^{1}$ M. Lühr ${ }^{2} \cdot$ C. Hagl ${ }^{2}$
}

${ }^{1}$ Klinik für Thorax- und Kardiovaskuläre Chirurgie, Westdeutsches

Herzzentrum Essen, Universitätsklinikum Essen

${ }^{2}$ Herzchirurgische Klinik und Poliklinik, Ludwig-Maximilians-Universität, Klinikum Großhadern, München

\section{Neue Rubrik „\#Leben“ stellt sich vor}

gründe uns jedoch allerdings oft unbekannt sind.

Entsprechend dem Grundgedanken wird die Rubrik primär von jungen Kollegen des Forums gestaltet. Die Aufgabe eines Mentors besteht lediglich darin, beratend zu agieren. Für das Junge Forum übernehmen diese Aufgabe federführend Daniel-Sebastian Dohle (Essen) und in Vertretung Maximilian Lühr (München). Christian Hagl (München) wird die Kollegen beratend unterstützten.

In dem aktuellen Beitrag von Placke $u$. Winter wird der aktuelle Stand der Batterieforschung unter die Lupe genommen. Die daraus resultierenden Zukunftsaussichten - auch für medizinische Anwendungen - werden in interessanter Weise erläutert und erlauben bereits jetzt einen spannenden Ausblick auf mögliche Veränderungen in unserem speziellen Fachgebiet.

Weitere interessante Arbeiten von qualifizierten Autoren aus verschiedensten Fachgebieten werden ab der Ausgabe 03/2015 folgen und hoffentlich mit großem Interesse von Ihnen gelesen werden. Schließlich gibt es im (\#)Leben noch mehr als nur Chirurgie ...

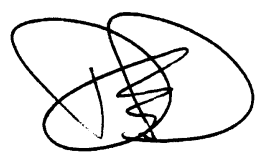

Daniel-Sebastian Dohle

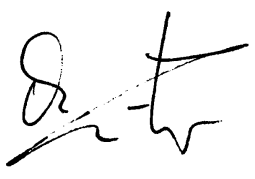

Maximilian Lühr

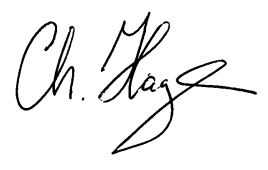

Christian Hagl

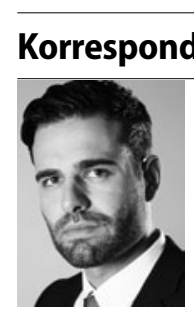

Klinik für Thorax- und Kardiovaskuläre Chirurgie, Westdeutsches Herzzentrum Essen, Universitätsklinikum Essen Hufelandstr. 55, 45147 Essen DS.Dohle@uk-essen.de

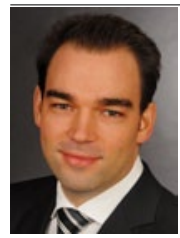

Dr. M. Lühr

Herzchirurgische Klinik und Poliklinik, Ludwig-MaximiliansUniversität, Klinikum Großhadern Marchioninistr. 15, 81377 München mail@dr-luehr.net

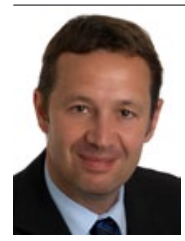

Prof. Dr. C. Hagl

Herzchirurgische Klinik und Poliklinik, Ludwig-MaximiliansUniversität, Klinikum Großhadern Marchioninistr. 15, 81377 München christian.hagl@ med.uni-muenchen.de

Interessenkonflikt. M. Lühr, C. Hagl und D.-S. Dohle geben an, dass kein Interessenkonflikt besteht. zen unseres Faches weit hinausgehen. Faszinierende technische Verfahren, Errungenschaften, gesellschaftliche Trends, Naturphänomene und deren Zusammenhänge sollen präsentiert und die Lebensgeschichten interessanter Menschen erzählt werden. Themen, die uns im alltäglichen Leben begegnen, deren Hinter- 